\title{
Hemoptisis como manifestación inusual de aneurisma de aorta torácica
}

\author{
Hemoptysis as an unusual manifestation of thoracic aortic aneurysm
}

\author{
Jenny Carolina Salazar-Flórez', \\ Tania Paola Rodríguez-Cuadrado', \\ Diego Alejandro Rangel-Rivera ${ }^{2} y$ \\ Manuel Fernando Pardo-Galvis ${ }^{3}$
}

\begin{abstract}
Salazar-Flórez JC, Rodríguez-CuadradoTP, Rangel-Rivera DA, Pardo-Galvis MF. Hemoptisis como manifestación inusual de aneurisma de aorta torácica. Rev Soc Peru Med Interna.

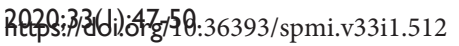

\begin{abstract}
RESUMEN
Los aneurismas aórticos corresponden a una dilatación focal de la pared de la arteria que supera el 50\% de su diámetro normal. La mayoría de los casos corresponden a la localización abdominal, siendo una pequeña proporción descritos a nivel de la aorta torácica. En este segmento, menos del 10\% de los pacientes experimentan síntomas e incluso, la ruptura puede constituir la primera manifestación. Se presenta el caso de una mujer octogenaria con hemoptisis e inestabilidad hemodinámica en la que se realizó el diagnóstico de aneurisma de aorta torácica a través angiotomografía contrastada para luego recibir manejo endovascular de manera exitosa. Dado el pronóstico de esta patología, es vital un adecuado uso de los estudios de imagen para un acertado diagnóstico radiológico que permita un enfoque terapéutico oportuno.
\end{abstract}

Palabras clave: aneurisma, hemoptisis, aorta torácica, endovascular, stent.

\section{ABSTRACT}

Aortic aneurysms correspond to a focal dilation of the artery wall that exceeds $50 \%$ of its normal diameter. Most cases correspond to the abdominal location, being a small proportion described at the level of the thoracic aorta. In this segment, less than $10 \%$ of patients experience symptoms and even rupture may constitute the first manifestation. It is presented an octogenarian woman with hemoptysis and hemodynamic instability in which the diagnosis of thoracic aortic aneurysm was made through contrasting angiotomography and then she

Médico general. Servicio de urgencias. Hospital Universitario de Santander, Bucaramanga, Colombia.

2 Médico residente de medicina interna. Grupo GERMINA, Universidad Industrial de Santander. Departamento de medicina interna. Hospital Universitario de Santander. Bucaramanga, Colombia.

3 Médico radiólogo. Departamento de radiología, Hospital Universitario de Santander. Bucaramanga, Colombia. receives a successfully endovascular management. Given the prognosis of this pathology, an adequate use of imaging studies is vital for a successful radiological diagnosis that allows for a timely therapeutic approach.

Keywords: aneurysm, hemoptisis, thoracic aorta, endovascular, stent.

\section{INTRODUCCIÓN}

Los aneurismas aórticos (AA) corresponden a una dilatación focal de la pared arterial que supera el $50 \%$ de su diámetro normal. ${ }^{1}$ A pesar de que el rango varía dependiendo del segmento arterial, edad, talla y género, se considera un valor normal entre 1,5 a $2,4 \mathrm{~cm}$. Por lo tanto, un registro superior a los $3 \mathrm{~cm}$ es criterio diagnóstico de dilatación aneurismática. ${ }^{2}$ La localización torácica corresponde al $12 \%$ de los casos de AA. ${ }^{3}$

El aneurisma de aorta torácica (AAT) tiene una incidencia aproximada de 6 a 10 casos por cada 100000 habitantes, 
principalmente entre la sexta a séptima década de la vida, con una proporción 4:1 de relación hombre a mujer. ${ }^{1} \mathrm{La}$ localización más frecuente en los AAT es el segmento ascendente en un $50 \%$ de los casos, segmento descendente en el $40 \%$ y en el arco aórtico solo un 10\%, aproximadamente. La ateroesclerosis asociada a otros factores clásicos de riesgo cardiovascular, como el tabaquismo, la hipertensión arterial y la obesidad, son claves en el desarrollo de AAT descendente. Otras entidades descritas como contribuyentes incluyen la disección aórtica crónica, endocarditis bacteriana, sífilis y enfermedades autoinmunes. ${ }^{2}$ En los casos de AAT ascendente, se le han relacionado con procesos de necrosis quística de la media dada por pérdida de células musculares lisas y degeneración de fibras elásticas que favorecen el debilitamiento de la pared arterial.

Los AAT suelen ser asintomáticos a pesar un crecimiento promedio de $0,1 \mathrm{~cm} /$ año descrito de forma previa. ${ }^{4}$ Cuando presentan manifestaciones, estas suelen ser secundarias a una complicación como disección, ruptura o insuficiencia aórtica severa. ${ }^{1}$ La presentación habitual involucra un cuadro de inicio súbito, caracterizado por dolor torácico irradiado a espalda o región epigástrica y síncope, con inestabilidad hemodinámica. ${ }^{4}$ La hemoptisis como síntoma de AAT es muy rara.

Se presenta el caso de una mujer adulta mayor quien presentó hemoptisis masiva y con diagnóstico final de aneurisma de aorta torácica con fístula bronquial, para el cual recibió manejo endovascular de forma exitosa.

\section{REPORTE DEL CASO}

Mujer de 82 años con antecedente de hipertensión arterial, artrosis de cadera bilateral y exposición al humo de segunda mano durante más de 20 años. Ella recibía manejo con losartán, metoprolol, hidroclorotiazida, acetaminofén y colchicina. Ingresó al servicio de urgencias con historia de una semana de tos con expulsión de abundantes coágulos asociado a palpitaciones y malestar general. En la revisión por sistemas, se documentó un mes de evolución de dolor en la región torácica no relacionada con el esfuerzo y disnea en reposo asociada a tos con expectoración amarillenta, astenia y adinamia.

Al ingreso, la paciente se encontró con palidez mucocutánea, frecuencia cardíaca 115 latidos/min), temperatura de $37,1^{\circ} \mathrm{C}$, frecuencia respiratoria de 20 excursiones/min y $\mathrm{SatO}_{2}$ de $93 \%\left(\mathrm{FiO}_{2}\right.$ al $\left.21 \%\right)$. El resto de examen físico sin alteraciones significativas.

Sobre la hora 6 de observación hospitalaria, presentó un episodio de tos en salvas con posterior cuadro interpretado como hematemesis (volumen aproximado de $1500 \mathrm{cc}$ ) asociado a taquipnea con desaturación, signos de dificultad respiratoria e hipotensión por lo que requirió intubación endotraqueal.

Se realizaron exámenes que mostraron anemia severa normocítica normocrómica y bioquímica sanguínea normal. Dado la religión de la paciente (testigo de Jehová) no se pudio usar hemoderivados, por lo cual el manejo de choque hipovolémico estuvo apoyado con solución salina, hierro parenteral y eritropoyetina.

La radiografía de tórax de ingreso fue descrita con aorta elongada, tortuosa con placa de ateroma a nivel del cayado, con mediastino ensanchado, sin consolidaciones en el parénquima pulmonar ni derrame pleural.

Dado la sospecha inicial de hemorragia de la vía digestiva alta se realizó una endoscopia que se reportó como normal. Se indicó una angiotomografía de tórax con protocolo para tromboembolia pulmonar y patología aórtica (Figuras 1 y 2). Se evidenció un aneurisma sacular en la porción inicial de la aorta descendente en su mayor parte constituido por trombo mural presentando compresión del parénquima pulmonar adyacente con atelectasia pasiva y contenido líquido hacia el espacio pleural. Se realizó un aortograma confirmando la presencia de úlcera penetrada en la aorta torácica descendente a aproximadamente $5 \mathrm{~cm}$ del nacimiento de la subclavia con un diámetro de $24 \mathrm{~mm}$. Se realizó un implante de endoprótesis $30 \mathrm{~mm}$ x $150 \mathrm{~mm} \times 30$ $\mathrm{mm}$ de forma exitosa.

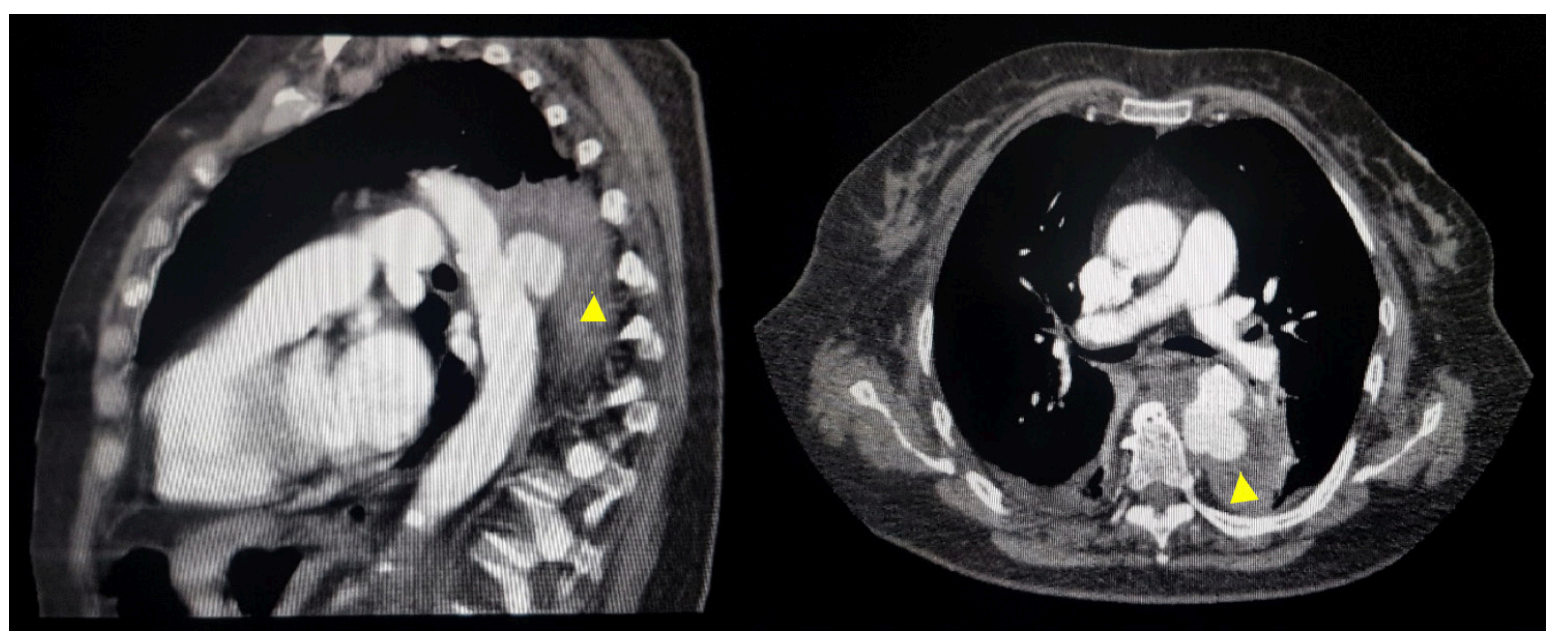

Figura 1. Tomografía de tórax con protocolo para aorta. A, B) Corte sagital y axial con aneurisma sacular en la porción inicial de la aorta descendente, con un cuello de 15 mm y un diámetro de 35 x 42 mm en sentido AP x transverso (flecha). Contenido líquido correspondiente a sangrado en el mediastino posterior (cabeza de flecha). 
Posterior al procedimiento, presentó paro cardiorrespiratorio con signos de taponamiento cardíaco que requirió pericardiocentesis con retorno a circulación espontánea. Se trasladó a la unidad de cuidados intensivos, con ecocardiograma transtorácico control posterior al procedimiento, sin evidencia de trastornos segmentarios de la contractilidad, fracción de eyección ventricular izquierda de $60 \%$ sin signos de taponamiento cardiaco.

La evolución clínica fue satisfactoria, el egreso hospitalario al día 20 de estancia y control ambulatoria a los 30 días con clase funcional NYHA II/IV, sin manifestaciones respiratorias o hemorrágicas.

\section{DISCUSIÓN}

La hemoptisis masiva representa menos del 2\% de los casos de hemoptisis y la mayoría están relacionados con casos de tuberculosis, neoplasias malignas, bola fungosa o comunicaciones anormales de la vía aérea con aneurismas de la aorta. ${ }^{5}$ El enfoque clínico de esta entidad es esencial dado la elevada mortalidad reportada en la literatura y que oscila entre el $38-50 \%$ de los casos. ${ }^{6}$

Los AAT son poco comunes en relación con el espectro completo de la enfermedad cardiovascular. ${ }^{7}$ La gran mayoría de los AAT son silenciosos y la ruptura constituye el primer síntoma en muchas ocasiones. ${ }^{8}$ Debido a la compresión que genera el aneurisma sobre las estructuras vecinas, se evidencia dolor que puede manifestarse a nivel cervical, torácico o abdominal. Cuando la vía aérea es la afectada, el paciente puede presentar sibilancias, neumonía recurrente, tos, disnea, hemoptisis o atelectasias, características presentes en el caso reportado. ${ }^{9}$

La hemoptisis es una manifestación muy rara de AAT y ocurre por la penetración dentro del árbol respiratorio. Con el tiempo, el crecimiento del aneurisma desencadena una respuesta inflamatoria y necrosis en la pared bronquial que determina la debilidad y formación de fístulas, lo que explica el alto riesgo de sangrado masivo que conduce a la muerte. ${ }^{10}$ En los pacientes con ruptura aórtica manifiesta hay una progresión típicamente rápida de la inestabilidad hemodinámica asociado a insuficiencia respiratoria, como sucedió en el caso presentado. ${ }^{7}$

El principal problema diagnóstico consiste en mantener un alto índice de sospecha de enfermedad aneurismática y conocer sus presentaciones. En particular, la disección aórtica se ha denominado "el gran imitador" porque puede producir síntomas relacionados con órganos de la vecindad $\mathrm{y}$, de esta manera, las presentaciones de disección aórtica son tan variadas y se pueden confundir con una amplia gama de otras enfermedades. ${ }^{8}$

Dentro del abordaje diagnóstico, la radiografía de tórax es normal en el $20 \%$, ensanchamiento del mediastino en el $50 \%$, contorno aórtico anormal en el $20 \%$, derrame pleural en el $8 \%$ y cardiomegalia por derrame pericárdico en menos del 5\%. ${ }^{4}$ En la mayoría de los centros, una tomografía de tórax con contraste será el procedimiento diagnóstico de elección dado su mayor accesibilidad. ${ }^{7}$ Si se dispone de resonancia magnética de forma rápida, esta es preferible ya que no hay radiación ni es necesario inyección de contraste. La aortografía no es un buen método inicial para el diagnóstico de un aneurisma pero se reserva para algunos escenarios como parte de la planeación en los casos que ya está decidida la intervención quirúrgica. ${ }^{11}$

El tamaño se constituye el principal marcador de riesgo de ruptura a 5 años. Es así que los aneurismas menores de 4 $\mathrm{cm}$ tienen un riesgo menor a $1 \%$; entre 4 a $5,9 \mathrm{~cm}$ un $16 \%$; $\mathrm{y}$, mayor o igual a $6 \mathrm{~cm}$ el riesgo es de $31 \% .^{2}$ Otros factores de riesgo que se han identificado incluyen dislipidemia, antecedente familiar de disección aortica o AAT, sexo femenino, tabaquismo, hipertensión arterial, edad avanzada y enfermedad pulmonar obstructiva crónica. ${ }^{12}$ Estas últimas cinco condiciones estuvieron presentes en nuestra paciente. La intervención quirúrgica es el tratamiento de elección en la mayoría de los pacientes. ${ }^{13}$ En la última década, la reparación aórtica endovascular torácica (TEVAR) aparece como una modalidad de tratamiento emergente que ha revolucionado el enfoque de los diversos tipos de enfermedades de la aorta en la zona torácica. ${ }^{14}$ Es así que el manejo endoluminal es una opción efectiva y menos invasiva que puede ser usado en pacientes no candidatos a procedimiento abierto, sea por el riesgo quirúrgico, edad, comorbilidades, esperanza de vida, entre otras. ${ }^{13,15}$

Como regla general, el manejo de los AAT pequeños asintomáticos es conservador basado en el control de los factores de riesgo con el objetivo de disminuir el efecto deletéreo en la pared y con ello el aumento progresivo de tamaño y el riesgo de ruptura. Algunos factores que pueden indicar la necesidad de corrección incluyen la insuficiencia aórtica severa, la disección aortica aguda tipo A (clasificación Stanford), síntomas atribuibles, diámetro mayor a $55 \mathrm{~mm}$ en aorta ascendente y arco aórtico, $77 \mathrm{~mm}$ en aorta descendente y $45 \mathrm{~mm}$ en paciente con antecedente de cirugía valvular aortica. ${ }^{2}$ No obstante, se debe tener claro que el beneficio de la intervención supere el riesgo propio del procedimiento antes de proponerlo al paciente. En conclusión, es infrecuente la presencia de un aneurisma de aorta torácica como causa de hemoptisis que amenaza la vida. La alta sospecha de esta patología en el escenario correcto permite detectar y tratar a tiempo esta condición cuya evolución clínica natural se corresponde con alta mortalidad. Es necesario reconocer las diferentes manifestaciones de una enfermedad que puede simular múltiples patologías, reconocer las imágenes iniciales sugestivas y elegir correctamente las ayudas diagnósticas, dado que el pronóstico sombrío se puede modificar con una oportuna intervención terapéutica.

\section{REFERENCIAS BIBLIOGRÁFICAS}

I. Goldfinger JZ, Halperin JL, Marin ML, Stewart AS, Eagle KA, Fuster V.Thoracic aortic aneurysm and dissection. J Am Coll Cardiol. 2014;64(I6): 1725-39.

2. Vega J, Gonzalez D, Yankovic W, Oroz J, Guaman R, Castro N. Aneurismas de la aorta torácica: Historia natural, diagnóstico y tratamiento. Rev Chil Cardiol. 20 I4;33:I27-35.

3. Ramírez J, Pozo M.Aneurisma de la aorta abdominal: controversias y tendencias en su diagnóstico y manejo. Rev Colomb Cirugía. 2010;25:323-31. 
4. Ramanath VS, Oh JK, Sundt TM, Eagle KA. Acute aortic syndromes and thoracic aortic aneurysm. Mayo Clin Proc. 2009;84(5):465-8I.

5. Jean-Baptiste E. Clinical assessment and management of massive hemoptysis. Crit Care Med. 2000;28(5): I642-7.

6. Khalil A, Fedida B, Parrot A, Haddad S, Fartoukh M, Carette M-F. Severe hemoptysis: From diagnosis to embolization. Diagn Interv Imaging. 20I5;96(7-8):775-88.

7. Hoel AW.Aneurysmal disease. Surg Clin North Am. 2013;93(4):893910.

8. Elefteriades JA. Thoracic Aortic Aneurysm: Reading the enemy's playbook. Curr Probl Cardiol. 2008;33(5):203-77.

9. Fleig A, Seitz K. Das thorakale Aortenaneurysma. Ultraschall der Medizin. Eur J Ultrasound. 2010;3 I (02): I22-43.

I0. Tanaka H, Yano M, Kuwabara C, Kume A, Tamura Y, Murakami $M$, et al. Massive hemoptysis due to the rupture of thoracic aortic aneurysm caused by leukemic cell infiltration in a patient with chronic myelomonocytic leukemia. J Clin Med Res. 2019; I I(2):।4550
II. Booher AM, Eagle KA. Diagnosis and management issues in thoracic aortic aneurysm. Am Heart J. 20 I I; I62(I):38-46.el.

12. Kim JB, Kim K, Lindsay ME, MacGillivray T, Isselbacher EM, Cambria RP, et al. Risk of rupture or dissection in descending thoracic aortic aneurysm. Circulation. 2015;132 (I7):1620-9.

13. Salameh MJ, Black JH, Ratchford EV.Thoracic aortic aneurysm. Vasc Med. 20I8;23(6):573-8.

14. Quintas A, Bastos Gonçalves F, Rodrigues H, Ferreira R, Oliveira $\mathrm{N}$, Rodrigues $\mathrm{G}$, et al. Tratamento endovascular de patologia da aorta torácica: experiência institucional. Angiol e Cir Vasc. 2016;12(I):3-II.

15. Tigkiropoulos K, Stavridis K, Lazaridis I, Saratzis N. Endovascular repair of aortobronchial fistula due to saccular aneurysm of thoracic aorta. Case Rep Vasc Med. 20I7;20 I 7:I-4.

\section{CORRESPONDENCIA:}

Correo electrónico: diegorangelrivera@gmail.com

FECHA DE RECEPCIÓN: 18-02-2020.

FECHA DE ACEPTACIÓN: 09-03-2020. 